\title{
MicroRNA-183 Acts as a Tumor Suppressor in Human Non-Small Cell Lung Cancer by Down-Regulating MTA1
}

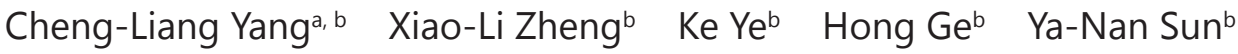 \\ Yu-Fei Lu ${ }^{\mathrm{b}}$ Qing-Xia Fan ${ }^{\mathrm{a}}$ \\ aDepartment of Oncology, The First Affiliated Hospital of Zhengzhou University, Zhengzhou, \\ bDepartment of Radiation Oncology, Affiliated Cancer Hospital of Zhengzhou University, Henan Cancer \\ Hospital, Zhengzhou, China
}

\section{Key Words}

Non-small cell lung cancer • MicroRNA-183 - MTA1 gene • Epithelial-mesenchymal transition - Invasion • Migration

\begin{abstract}
Backgrounds/Aims: MicroRNAs (miRs) often contribute to the progression of non-small cell lung cancer (NSCLC) via regulation of mRNAs that are involved in lung homeostasis. We conducted a study aimed at exploring the roles of miR-183 in the proliferation, epithelialmesenchymal transition (EMT), invasion and migration of human NSCLC cells via targeting MTA1. Methods: NSCLC and adjacent normal tissues were collected from 194 patients with NSCLC. Positive expression of MTA1 protein was detected by immunohistochemistry. The highest levels of expression of miR-183 were detected using RT-qPCR in SPC-A-1 cells, which were selected and assigned to the following groups: blank, negative control (NC), miR-183 mimic, miR-183 inhibitor, siRNA-MTA1, and miR-183 inhibitor + siRNA-MTA1. The expression of miR-183 and the mRNA and protein expression of MTA1, E-cadherin, Vimentin, Snail, PCNA, $\mathrm{Bax}$ and $\mathrm{BCl}-2$ in tissues and transfected cells were measured using RT-qPCR and western blot analysis. Cell proliferation, apoptosis, migration and invasion were evaluated by CCK-8, flow cytometry, scratch tests and Transwell assays. Tumor xenografts were conducted in nude mice to determine tumor growth. Results: SPC-A-1 cells with the highest levels of miR-183 expression were selected. Compared with adjacent normal tissues, the expression of miR183 and the mRNA and protein expression of E-cadherin and Bax were decreased in NSCLC tissues, while mRNA and protein expression of MTA1, Vimentin, snail, PCNA and Bcl-2 were increased. MiR-183 was over-expressed in the miR-183 mimic group and under-expressed in the miR-183 inhibitor and miR-183 inhibitor + siRNA-MTA1 groups. In the miR-183 mimic and siRNA-MTA1 groups, the mRNA and protein expression of E-cadherin and Bax, as well as cell apoptosis, were enhanced, while the expression levels of MTA1, Vimentin, snail, PCNA and $\mathrm{Bcl}-2$ mRNA and protein, cell proliferation, migration, invasion and tumor growth were reduced relative to the blank and NC groups. The miR-183 inhibitor group exhibited an opposite trend. Conclusion: Our study indicates that miR-183 down-regulates MTA1 to inhibit the proliferation, EMT, migration and invasion of human NSCLC cells.




\section{Introduction}

Lung cancer is one of the most common malignancies, and it is also a leading cause of cancer death around the world, causing more deaths every year than breast, prostate and colon cancer combined [1,2]. Clinically, non-small-cell lung cancers (NSCLCs) account for more than $80 \%$ of lung cancers, including adenocarcinomas, squamous-cell and large-cell carcinomas [3]. NSCLC is a heterogeneous tumor [4] with multiple risk factors that include performance status, bone metastases of leukopenia, age, albumin levels, body surface area (BSA), neutropenia, gender, and serum creatinine concentration in thrombocytopenia [5]. In NSCLC, the estimated 5-year survival is only 16\%. Although surgery is commonly regarded as the optimal treatment, approximately $30 \%$ of patients qualify for undergoing potentially curative resection, and another $20 \%$, mainly those suffering with locally advanced disease, usually choose to undergo radical thoracic radiotherapy treatments, with or without chemotherapy. The remaining $50 \%$ of patients, most of whom had late-stage or metastatic disease, generally chose palliative treatments [6]. Thus, this demonstrates that there is a need for newer and more accurate predictors to help provide a better diagnosis and prognosis for NSCLC. MicroRNAs (miRs), as small non-coding RNA molecules, have been demonstrated to play a vital role in cancer [7]. It has been found that several miRs, such as miR-130a, miR-708 and miR-183, are involved in NSCLC [8-10].

MiR-183 is viewed as a potential inhibitor of metastasis, and the expression of miR-183 is negatively associated with the metastatic potential of lung cancer cells [11]. Moreover, up-regulated miR-183-3p was reported to be a potent prognostic marker for lung cancer in female non-smokers [12]. In addition, a previous study demonstrated that miR-183 could repress epithelial-mesenchymal transition (EMT) and invasion of human pancreatic cancer cells by inhibiting the expression of MTA1 [13]. MTA1 is the founding member of the MTA family of coregulatory factors in transcriptional programs, and plays an integral role in nucleosome remodeling and histone deacetylation (NuRD) [14]. Overexpression of MTA1 is significantly associated with tumor progression and poor prognosis in NSCLC cells [15], and it has been demonstrated that the overexpression of MTA1 protein is common in early-stage NSCLC and is obviously related to tumor angiogenesis and poor survival [16]. A previous study demonstrated that MTA1 plays a key role in the regulation and mediation of the invasive phenotypes of lung cancer cells, and it has been reported that this regulation functions by altering the expression of miR [14]. However, the mechanism by which MTA1 protein regulates the malignant progression of NSCLC is not well understood [17].

Although we have some understanding of the occurrence and treatment of tumors, especially for NSCLC, our understanding remains limited. There also remains a pressing need to develop strategies that target tumor cells and their genetic features [18]. In this study, our goal was to evaluate the effects of miR-183 on proliferation, EMT, migration and invasion of human NSCLC cells by its regulation of the expression of MTA1.

\section{Materials and Methods}

\section{Ethical statement}

This study was approved by the Ethics Committee of the First Affiliated Hospital of Zhengzhou University. Patients and legal guardians were informed of the study subject, and they signed informed consent forms.

\section{Study subjects}

A total of 194 patients with NSCLC that received surgical treatments in the First Affiliated Hospital of Zhengzhou University from September 2012 to September 2015 were selected for the study. Tumor tissues and adjacent normal tissues (taken approximately $5 \mathrm{~cm}$ from the lung cancer tissues) were collected. All tissues were stored at $-80^{\circ} \mathrm{C}$ and were confirmed as NSCLC. Inclusion criteria were as follows: (1) Patients diagnosed with NSCLC according to diagnostic standards [19] by cytologist or pathologist and were unable 


\section{Cellular Physiology Cell Physiol Biochem 2018;46:93-106 \begin{tabular}{l|l|l}
\hline and Biochemistry 10.1159/000488412 & $\begin{array}{l}\text { (c) 2018 The Author(s). Published by S. Karger AG, Basel } \\
\text { www.karger.com/cpb }\end{array}$
\end{tabular}}

Yang et al.: Role of MiR-183 and MTA1 in NSCLC

or unwilling to undergo surgery; (2) The age range was 18 - 75 years, the Karnofsky score was greater than 70 , and the expected survival time was more than 6 months using an objective measurable lesion index. The routine blood parameters, liver, kidney functions and electrocardiographs (ECG) were normal prior to receiving treatment. The exclusion criteria were as follows: (1) Patients diagnosed with NSCLC by cell pathology alone (lung biopsy cytology, bronchoscopy brush, etc.); (2) NSCLC patients diagnosed without histopathology of primary tissue tumors.

\section{Cell culture, grouping and transfection}

NSCLC cell lines (H1299, SPC-A-1, 95D and A549) were purchased from Shanghai Institutes for Biological Sciences, Chinese Academy of Sciences and inoculated in medium containing 10\% fetal bovine serum (FBS), RPMI 1640 medium, $100 \mathrm{U} / \mathrm{mL}$ penicillin and $50 \mathrm{mg} / \mathrm{L}$ streptomycin and then cultured in an incubator with $5 \% \mathrm{CO}_{2}$ at $37^{\circ} \mathrm{C}$. The medium was replaced every $24 \sim 48 \mathrm{~h}$, and the cells were treated with $0.25 \%$ trypsin and passaged. The third passage was selected for the study. The expression of MiR183 in the cell lines was determined using RT-qPCR, and the high-expression cell line was selected for further study. The cells were divided into blank, negative control (NC), miR-183 mimic, miR-183 inhibitor, siRNA-MTA1, and miR-183 inhibitor + siRNA-MTA1 groups. Cells were seeded in 6-well cell culture plates prior to transfection. When cell confluence reached approximately 50\%, the human NSCLC cell lines were transfected transiently using Lipofectamine ${ }^{\mathrm{TM}} 2000$ (11668027, Invitrogen, USA). The lyophilized powders from the blank, NC, miR-183 mimic, siRNA-MTA1, miR-183 inhibitor and miR-183 inhibitor + siRNA-MTA1 (Invitrogen, Car, Cal, USA) were centrifuged and dissolved in water without RNase. A volume of $5 \mu \mathrm{l}$ of Lipofectamine 2000 was diluted with $250 \mu \mathrm{l}$ of serum-free medium (Opti-MEM, Gibco, Invitrogen, USA), incubated for $5 \mathrm{~min}$ at room temperature, swirled gently to mix evenly and then incubated for 20 mins at room temperature. The mixture was then added to the cell culture wells and incubated at $37^{\circ} \mathrm{C}$ with $5 \% \mathrm{CO}_{2}$. After $6 \sim 8 \mathrm{~h}$, the mixture was replaced by complete medium and the cells were incubated for $24 \sim 48 \mathrm{~h}$.

\section{Dual luciferase reporter gene assay}

The target genes of miR-183 were analyzed using the website http://www.microrna.org to verify that MTA1 is the direct target gene of miR-183. The target and mutant sequences were designed according to the miR-183-binding sequence in the 3'-untranslated region (UTR) of the MTA1 mRNA. The target sequence was synthesized with Xho $I$ and Not $I$ restriction sites at each end. The synthesized fragments were cloned into PUC57. After identification of positive clones and recombinant plasmids using DNA sequencing, the recombinant plasmid was transformed into Escherichia coli DH5 $\alpha$ cells using psiCHECK-2 for plasmid amplification. The plasmids were extracted according to the Omega kit instructions. Cells were inoculated into 6 -well plates $\left(2 \times 10^{5}\right.$ cells/well). Adherent cells were transfected and cultured for $48 \mathrm{~h}$, then collected. The Dual-Luciferase reporter gene assay was used according to the kit instructions (Genecopoeia, Guangzhou, China) to detect luciferase activity in MTA1 3'-UTR cells that were affected by miR-183. Fluorescence-intensity was measured using a Glomax20/20 luminometer fluoroscope (Promega, Beijing, China).The experiments in each group were repeated 3 times.

\section{Immunohistochemistry}

Tissues were fixed with $10 \%$ formalin, dehydrated with graded ethanol, embedded with paraffin and then cut into $5 \mu \mathrm{m}$ sections. Sections were baked at $60^{\circ} \mathrm{C}$ for $1 \mathrm{~h}$ and washed 3 times with xylene (10 min each time) in order to dewax. After dewaxing, the sections were dehydrated in graded ethanol (95\%, $80 \%$ and $75 \%$ ) for $1 \mathrm{~min}$ each, washed with tap water for $1 \mathrm{~min}$ and then incubated $30 \mathrm{~min}$ at $37^{\circ} \mathrm{C}$ with $3 \%$ hydrogen peroxide. After washing with $1 \times$ phosphate-buffered saline (PBS), sections were heated in $0.01 \mathrm{M}$ citrate buffer at $95^{\circ} \mathrm{C}$ for $20 \mathrm{~min}$, then cooled to room temperature and washed with $1 \times$ PBS. The sections were blocked for $10 \mathrm{~min}$ by the addition of $10 \%$ goat serum at $37^{\circ} \mathrm{C}$, and then, rabbit anti-human MTA1 polyclonal antibody (1 : 2000, ab71153, Abcam, Cambridge, MA, USA) was added and incubated overnight at $4^{\circ} \mathrm{C}$. The sections were washed with $1 \times$ PBS for 2 min and then incubated for 30 min at room temperature after the addition of goat anti-rabbit serum $(1: 1000$, DF7852, Shanghai Yaoyun Biotechnology Co., Ltd., Shanghai, China). The sections were then labeled with horseradish peroxidase and treated with 3, 3'-diaminobenzidine (DAB) (CAS: 7411-49-6, Suzhou Yacoo Chemical Reagent Co., Ltd, Suzhou, Jiangsu, China). Hematoxylin (PT001, Shanghai Bogoo Biological Technology Co. Ltd., Shanghai, China) was used as the counterstain, and the sections were then mounted. In each slice, 10 visual fields were randomly selected 
under the microscope (CX31, Beijing Chang Heng Rong Technology Co. Ltd., Beijing, China). A total of 100 cells were counted in each field $(400 \times)$ and the percentage of positive cells was calculated. The degree of staining was classified as follows: no color (negative), bright yellow (weakly positive), yellow (moderately positive) and brown (strongly positive) [20].

Reverse transcription quantitative polymerase chain reaction ( $R T-q P C R$ ) assays

Total RNA was extracted from NSCLC and adjacent normal tissues using a miRNeasy Mini Kit (217004, Qiagen GmbH, Hilden, Germany). Primers were designed and then synthesized by Takara (TaKaRa Biotechnology Co. Ltd., Tokyo, Japan). Primer sequences of RT-qPCR are shown in Table 1. The complementary deoxyribonucleic acid (cDNA) was synthesized using a PrimeScript RT kit (RR036A, TaKaRa Biotechnology Co. Ltd., Tokyo, Japan) according to the instructions, and the total volume of the system was $10 \mu \mathrm{l}$. The reaction conditions were as follows: pre-denaturation at $95^{\circ} \mathrm{C}$ for $30 \mathrm{~s}$, and then a $5 \mathrm{~s}$ denaturation at $95^{\circ} \mathrm{C}$ followed by annealing and elongation for $30 \mathrm{~s}$ at $60^{\circ} \mathrm{C}$ for 40 cycles. The reaction liquid was used for RT-qPCR. The RT-qPCR was conducted according to the instructions in the SYBR $®$ Premix Ex Taq ${ }^{\mathrm{TM}}$ II kit (RR820A, TaKaRa Biotechnology Co. Ltd., Tokyo, Japan). The PCR system was as follows: $25 \mu \mathrm{l}$ of SYBR® Premix Ex Taq ${ }^{\mathrm{TM}}$ II $(2 \times), 2 \mu \mathrm{l}$ of forward primer, $2 \mu \mathrm{l}$ of reverse primer, $1 \mu \mathrm{l}$ of ROX Reference Dye $(50 \times), 4 \mu \mathrm{l}$ of DNA template and $16 \mu \mathrm{l}$ of doubly distilled water $\left(\mathrm{ddH}_{2} \mathrm{O}\right)$. An ABI 7500 PCR machine (7500, ABI, USA) was used to conduct the RT-qPCR. The reaction conditions were as follows: predenaturation at $95^{\circ} \mathrm{C}$ for $30 \mathrm{~s}, 40$ cycles of denaturation at $95^{\circ} \mathrm{C}$ for $5 \mathrm{~s}$, and annealing and elongation at $60^{\circ} \mathrm{C}$ for $30 \mathrm{~s}$. U6 was used as the internal reference for miR-183 expression, and glyceraldehyde-3-phosphate dehydrogenase (GAPDH) was a reference for the expression of MTA1, E-cadherin, Vimentin, Snail, PCNA, Bax and Bcl-2. The reliability of the PCR was evaluated using solubility curves. The Ct value was obtained (knee of amplification dynamic curve), and the relative expression of the target gene was analyzed using the $2^{-\Delta \Delta \mathrm{Ct}}$ method, where $\Delta \Delta \mathrm{Ct}=\Delta \mathrm{Ct}_{\text {tumor group }}-\Delta \mathrm{Ct}_{\text {normal group }}, \Delta \mathrm{Ct}=\mathrm{Ct}_{\text {target gene }}-\mathrm{Ct}_{\text {internal reference' }}$, and the relative transcription level of target gene mRNA $=2^{-\Delta \Delta \mathrm{Ct}}[21]$. (This assay was also performed in the cell experiments).

\section{Western blot analysis}

Liquid nitrogen was added to the fresh tissues. The samples were ground into a fine powder, then reacted in protein lysis buffer and placed in an ice bath. The samples were shaken slowly for $1 \mathrm{~h}$ and complete lysis was achieved. After centrifugation at $12,000 \mathrm{rpm}$ for $20 \mathrm{~min}$ at $4^{\circ} \mathrm{C}$, the supernatant was collected for analysis. The protein concentrations of each sample were determined and the samples were adjusted to the same concentration. Proteins were separated using sodium dodecyl sulfate-polyacrylamide gel electrophoresis (SDS-PAGE) (10\% gels, 20050227, Beyotime Biotechnology, Shanghai, China), followed by transfer of the proteins onto a nitrocellulose membrane which was then blocked with 5\% BSA at room temperature for $1 \mathrm{~h}$. The proteins were then incubated overnight with first antibodies at $4^{\circ} \mathrm{C}$. The first antibodies were as follows: rabbit anti-human MTA1 (1 : 2000, ab71153); E-cadherin antibody (1: 50, ab1416); Vimentin antibody (1 : 5000, ab92547); Snail antibody ( $:$ 500, ab53519); PCNA antibody (1: 5000, ab97080); Bax antibody (1: 2000, ab32503) and Bcl-2 antibody ( $1: 2000$, ab32503). The antibodies were purchased from Abcam, Cambridge, MA, USA. After washing with Tris-buffered saline containing Tween-20 (TBST) 5 times for 5 min each time, the membranes were incubated with horseradish peroxidase-labeled goat anti-rabbit IgG antibody (1 : 1000, Wuhan Boster Biological Technology co. Ltd., Wuhan, China) as the second antibody at $37^{\circ} \mathrm{C}$ for $1 \mathrm{~h}$, then washed 3 times with PBS for 5 min each time. The membranes were immersed in an electrochemiluminescence (ECL) solution (WBKLS0500, Pierce, Rockford, IL, USA), observed in a darkroom, and pictures were taken. IPP7.0 software (Media Cybernetics, Singapore) was 


\section{Cellular Physiology Cell Physiol Biochem 2018;46:93-106 \begin{tabular}{l|l|l} 
and Biochemistry 10.1159/000488412 & $\begin{array}{l}\text { D } 2018 \text { The Author(s). Published by S. Karger AG, Basel } \\
\text { www.karger.com/cpb }\end{array}$
\end{tabular}}

Yang et al.: Role of MiR-183 and MTA1 in NSCLC

used for quantitative analysis. The ratio of gray values between bands of MTA1, E-cadherin, Vimentin, Snail, PCNA, Bax, Bcl-2 and GAPDH were calculated and presented as the relative protein expression (this method was also applicable for cell experiments).

\section{Cell Counting Kit-8 (CCK-8)}

After $24 \mathrm{~h}$ of transfection, the cells were washed 2 times with PBS and then digested with $0.25 \%$ trypsin to obtain single cell suspensions. The cells were inoculated at a density of $3 \times 10^{3} \sim 6 \times 10^{3} /$ cells/well (200 ul/ well) in a 96-well plate after counting, and 6 parallel wells were established for each group. The cells were cultured for 24, 48 and $72 \mathrm{~h}$. Then, $10 \mu \mathrm{l}$ of CCK-8 reagent (Sigma, St Louis, MO, USA) was added to each well, and the cells were then cultured for $2 \mathrm{~h}$. An Enzyme-linked immunosorbent assay (ELISA) (NYW96M, Beijing Noah Instrument Co., Ltd., Beijing, China) was employed and the optical density (OD) value was measured at $570 \mathrm{~nm}$. Each experiment was conducted in triplicate. Cell survival curves were drawn using time for the abscissa and OD values for the ordinate.

\section{Flow cytometry}

Cell cycles were analyzed by staining with propidium iodide (PI). After $48 \mathrm{~h}$ of transfection, the cells were collected and fixed at $4^{\circ} \mathrm{C}$ in $75 \%$ ice-cold ethyl alcohol overnight. The cells were then centrifuged, the supernatant was removed, and the cells were washed 2 times with PBS containing 1\% FBS and then suspended in $400 \mu \mathrm{l}$ of binding buffer. After the addition of $50 \mu \mathrm{l}$ of RNase A (Sigma, St Louis, MO, USA), the cells were incubated for $30 \mathrm{~min}$ at $37^{\circ} \mathrm{C}$. The cells were placed in the dark with $50 \mu \mathrm{l}$ of PI $(50 \mathrm{mg} / \mathrm{L}$, Sigma, St Louis, MO, USA) and incubated for $30 \mathrm{~min}$ at room temperature. Cell cycle analyses were performed using flow cytometry. The DNA content distribution curve was obtained by measuring the DNA content of each cell in the cell population. The experiment was repeated 3 times. Cell cycle distribution curves were drawn using the number of effective cells as the ordinate and the DNA content as the abscissa.

Annexin V FITC/PI double staining was conducted to determine cell apoptosis. Cells were inoculated in 6-well plates $\left(2 \times 10^{5}\right.$ cells/well $)$ after $48 \mathrm{~h}$ of transfection. The cells were examined by double staining with $100 \mathrm{nmol} / \mathrm{L}$ Annexin V FITC/PI. After $72 \mathrm{~h}$ of transfection, the cells were washed with PBS at $4^{\circ} \mathrm{C}$ and then removed from the plates by digestion with trypsin. The cells were collected in $15 \mathrm{ml}$ centrifuge tubes and centrifuged at $800 \times \mathrm{g}$. After removing the supernatant and washing the cells twice with PBS, the cells were re-suspended with $500 \mu \mathrm{l}$ of Annexin V-FITC binding buffer and incubated with $5 \mu$ l of Annexin V-FITC solution and $5 \mu \mathrm{l}$ of PI for $15 \mathrm{~min}$ in the dark according to the instructions for the BD cell apoptosis detection kit I (Annexin V-HTC Apoptosis Detection Kit I, 556547). Cell apoptosis was evaluated using flow cytometry. In the apoptosis scatter diagram, the left lower quadrant represents normal cells (An-PI-) and the right lower quadrant represents early cell apoptosis (An+PI-). The right upper quadrant represents advanced cell apoptosis and necrosis (An-PI+), and the left upper quadrant represents damaged cells (An-PI+) that appear during cell collection. The percentage of early apoptotic cells relative to the total number of cells was expressed as the apoptosis rate. The experiment was repeated in triplicate.

\section{Scratch test}

Cells from each group were inoculated into 6-well plates and cultured in serum-free Dulbecco minimum essential medium (DMEM) after cells adhered to the wall. After the cells reached approximately $90 \% \sim 100 \%$ confluency, a $10 \mu \mathrm{l}$ pipette was used to perpendicularly scratch the bottom of the plate $(4$ $\sim 5$ /well), keeping the width of each scratch constant. The cells were then cultured in the incubator after washing three times with PBS. To measure the migratory response of the cells, an inverted microscope was used to observe and record images at 0 and $24 \mathrm{~h}$ after scratching. Each group was established in three wells, and the experiment was conducted in triplicate.

\section{Transwell assays}

A transwell chamber was placed into a 24-well plate. The surface of the upper chamber was covered with Matrigel diluent $(1: 8)$ and air-dried at room temperature. The cells in the control, blank, NC, miR-183 mimic, miR-183 inhibitor, miR-183 inhibitor + siRNA-MTA 1 and miR-183 mimic + siRNA-MTA1 groups were digested and washed with PBS two times and then re-suspended with RPMI1640 culture medium. The cell density was adjusted to $1 \times 10^{5} / \mathrm{mL}$. Transwell chambers were planted on the Matrigel. Cell suspensions $(200 \mu \mathrm{l})$ were added to each Transwell chamber in the upper layer, and RPMI 1640 containing 20\% FBS 
$(600 \mathrm{ul})$ was added in the lower layer. After culturing for $24 \mathrm{~h}$, the cells on the upper surface of the Transwell membrane were wiped off with a cotton swab, fixed with $4 \%$ paraformaldehyde for 15 min, stained with $0.5 \%$ crystal violet (prepared with methanol) for $15 \mathrm{~min}$ and then washed three times with PBS. A total of 5 fields $(200 \times)$ were randomly selected, and images were obtained using an inverted microscope (XDS-800 D, Shanghai Cai Kang Optical Instrument Co., Ltd., Shanghai, China). The average number of cells permeating the septum was recorded. Three wells were established for each group and the experiments were conducted in triplicate.

Tumor xenograft in nude mice

After $48 \mathrm{~h}$ of transfection, human NSCLC cells were collected and single cell suspensions were prepared. The cells were re-suspended in a mixture containing PBS and Matrigel $(1: 1)$. The concentration of the cell suspension was adjusted to $1 \times 10^{6}$ cells $/ 200 \mu \mathrm{l}$. The 18 enrolled nude mice were further classified into six groups as follows: blank, NC, miR-183 mimic, miR-183 inhibitor, siRNA-MTA1, and miR-183 inhibitor + siRNA-MTA1 groups, with 3 mice in each group. Nude mice were inoculated with NSCLC cells $\left(1 \times 10^{6}, 200\right.$ ul) under ether anesthesia. The mice were raised in the same environment and were observed every week. Tumor lengths and widths were recorded and the tumor volume was calculated according to the formula: tumor volume $=\left(\right.$ length $\times$ width $\left.{ }^{2}\right) / 2$. The nude mice were sacrificed on the thirty-fifth day, the tumors were dissected out, and 3 tumor samples were collected from each group.

\section{Statistical analysis}

SPSS 21.0 software (IBM Corp Armonk, NY, USA) was used to perform statistical analysis. Measurement data were displayed as the mean \pm standard deviation (SD). The differences between two different groups were analyzed using a $t$-test, and differences among multiple groups were analyzed by one-way analysis of variance (ANOVA). The level of significance was set at $p<0.05$.

\section{Results}

\section{A higher positive rate of MTA1 protein expression is observed in NSCLC tissues}

Initially, immunohistochemistry was performed to compare the positive rates of MTA1 protein expression in NSCLC and adjacent normal tissues. The results demonstrate that MTA1 in NSCLC tissue was located mainly in the cytoplasm in brownish yellow granules. Positive rates of MTA1 protein expression in adjacent normal tissues were $23.32 \%$ and $65.25 \%$ in NSCLC tissues. The difference between the two groups was statistically significant $(p<0.05)$ (Fig. 1). The results reveal that the positive rate of MTA1 protein expression is higher in NSCLC tissues than in the adjacent normal tissues.

Higher miR-183 expression and lower MTA1 mRNA expression are observed in NSCLC tissues

To investigate the expression of miR-183 and mRNA expression of MTA1 related genes, RT-qPCR was performed. The results are shown in Fig. 2. In comparison with adjacent normal tissues, miR-183 expression and mRNA expression of E-cadherin and Bax were significantly increased, while mRNA expression levels of MTA1, Vimentin, snail, PCNA and Bcl-2 were significantly decreased $(p<0.05)$. The results suggest that higher miR183 expression and lower MTA1 mRNA expression are observed in NSCLC tissues.

Higher expression of MTA1, Vimentin, Snail, PCNA and Bcl-2 proteins, and lower expression of E-cadherin and Bax proteins are observed in NSCLC tissues

Subsequently, western blot analysis was conducted to detect the

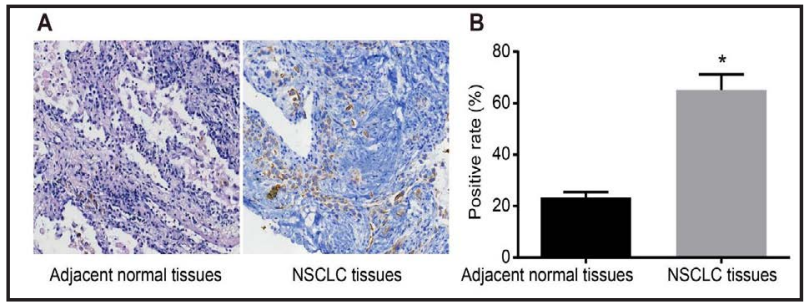

Fig. 1. Immunohistochemistry results reveal a higher positive rate of MTA1 protein expression in NSCLC tissues (A, $\times 200$ ). Note: NSCLC, non-small cell lung cancer; ${ }^{*}, \mathrm{p}<0.05$ compared with the adjacent normal tissues. 
expression of MTA1, Vimentin, Snail, PCNA, Bcl-2, E-cadherin and Bax proteins. As shown in Fig. 3, the results suggest that compared with adjacent normal tissues, the expression of MTA1, Vimentin, Snail, PCNA and Bcl-2 proteins increased significantly, while that of E-cadherin and Bax decreased significantly ( all $p<0.05$ ).

The highest expression of miR-183 is found in the SPC-A-1 cell line

Next, the expression of miR-183 in H1299, SPC-A-1, 95D and A549 NSCLC cell lines were detected by RT-qPCR in order to select the highest-expressing cell line. As shown in Fig. 4, miR-183 expression differed in the human NSCLC cell lines and was highest in the SPC-A-1 cell line $(p<0.05)$. Therefore, the SPC-A-1 cell line was selected for further study.

\section{MTA1 is a target gene of miR-183}

Based on the online tool http://www.microrna.org, there is a specific binding region between the MTA1 gene sequence and the miR-183 sequence, and MTA1 is the target gene of miR-183 (Fig. 5-A). The luciferase reporter gene assay verified that MTA1 was a target gene of miR-183 (Fig.

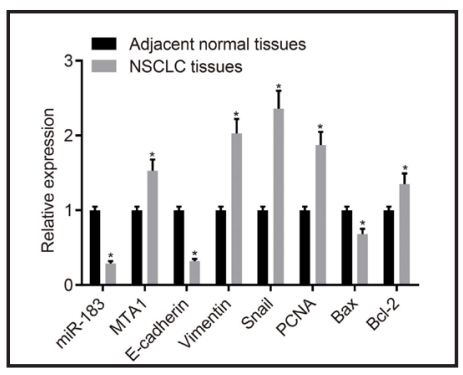

Fig. 2. RT-qPCR results reveal higher levels of miR-183 expression and lower levels of MTA1 mRNA expression in NSCLC tissues. Note: miR-183, microRNA-183; RT-qPCR, reverse transcription quantitative polymerase chain reaction; PCNA, proliferating cell nuclear antigen; NSCLC, non-small cell lung cancer; MTA1, metastasis-associated protein 1 ; *, $\mathrm{p}<0.05$ compared with adjacent normal tissues.
5-B). The results indicated that the luciferase activity in the miR-183 mimic group that was co-transfected with WtmiR-183/MTA1 was reduced compared to the NC group $(p<0.05)$, while the groups co-transfected with mutant 3'UTR exhibited no significant inhibition of luciferase activity ( $p>0.05$ ), suggesting that miR183 could bind specifically to MTA1. Therefore, MTA1 is a potential target gene for miR183.

Up-regulated miR-183 expression decreases MTA1 mRNA expression

To study the mechanisms and functions of miR-183 and MTA1 in NSCLC, their mRNA expression levels were measured in transfected cells in vivo using RT-qPCR. There was no significant difference in the mRNA expression between the blank and NC groups $(p>0.05)$. Compared with the blank and NC groups, the expression of mRNA for E-cadherin and Bax increased significantly in the miR-183 mimic and siRNAMTA1 groups, while that in the MTA1, Vimentin, Snail, PCNA and Bcl-2 groups decreased (all $p<0.05)$. The expression of miR-183 was increased in the miR-183 mimic group $(p<$ $0.05)$; however, no significant difference was observed in the siRNA-MTA1 group $(p>0.05)$. In the miR-183 inhibitor group, the expression levels of miR-183, E-cadherin and Bax mRNA 
declined, while the expression levels of MTA1, Vimentin, Snail, PCNA and Bcl-2 mRNA increased (all $p<0.05$ ). In the miR-183 inhibitor + siRNA-MTA1 group, miR-183 expression was reduced $(p<0.05)$, while no significant difference was observed in the expression of MTA1, Vimentin, Snail, PCNA and Bcl-2 mRNA $(p>0.05)$ (Fig. 6). These data suggest that overexpression of miR-183 could down-regulate the expression of MTA1 mRNA.

Up-regulation of miR183 expression decreases expression of MTA1 protein

To study the mechanisms and functions of miR-183 on the expression of MTA1 protein in NSCLC, the expression levels of MTA1, E-cadherin, Vimentin, Snail, PCNA, Bax and Bcl-2 proteins were measured in transfected cells in vivo using western blot analysis. The results of western blot analysis indicate no notable difference in the expression of these proteins between the blank and NC groups $(p>0.05)$. Compared with the blank and NC groups, the expression of E-cadherin and Bax proteins were significantly

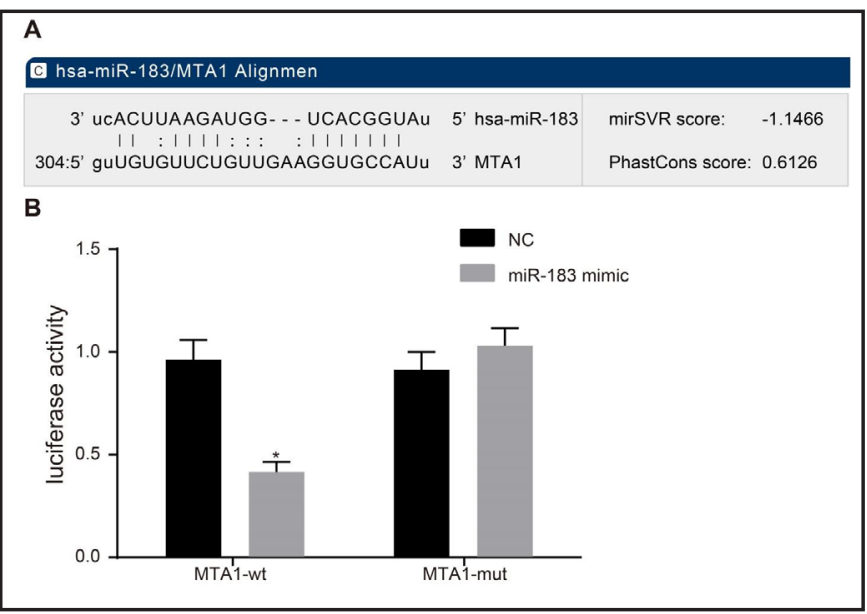

Fig. 4. RT-qPCR results indicate that the highest levels of miR183 expressions are found in the SPC-A-1 cell line. Note: MiR183, microRNA-183; RT-qPCR, reverse transcription quantitative polymerase chain reaction; NSCLC, non-small cell lung cancer; UTR, 3' untranslated region; *, p<0.05 compared with SPC-A-1 cell line.

Fig. 5. MTA1: the target gene of miR-183. Note: A, predicted binding sites of miR-183 on MTA1 3'UTR; $\mathrm{B}$, detection of luciferase activity using a luciferase reporter assay;

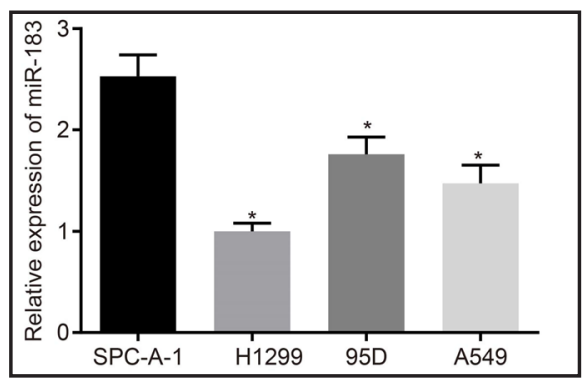
miR-183, microRNA-183; UTR, 3' untranslated region; MTA1, metastasis-associated protein 1 ; NC, negative control; *, $\mathrm{p}<0.05$ compared with the NC group. increased, while the expression of MTA1, Vimentin, Snail, PCNA and Bcl-2 proteins were decreased in the miR-183 mimic and siRNA-MTA1 groups (all $p<0.05$ ). The MiR-183 inhibitor group exhibited an opposite trend, and protein expression in the miR-183 inhibitor + siRNA-MTA1 group exhibited no significant difference $(p>0.05)$ (Fig. 7). These results suggest that overexpression of miR183 could down-regulate the expression of MTA1 protein.

\section{Up-regulation of miR-183 inhibits NSCLC cell proliferation}

In the following experiments, we investigated mainly the ability of miR-183 to influence the biological functions of NSCLC cells. The CCK-8 results indicate that the main effects of different groups, different time points, and interactions between groups and time points on cell proliferation were statistically significant $(p<0.05)$. An obvious difference in cell proliferation was not observed between the blank and the NC groups $(p>0.05)$. Compared with the blank and NC groups, the cell proliferation ability was significantly lower in the miR183 mimic and the siRNA-MTA1 groups, while it was higher in the miR-183 inhibitor group (all $p<0.05$ ). No significant difference in cell proliferation was observed among the miR-183 inhibitor + siRN-MTA1, blank and NC groups $(p>0.05)$ (Fig. 8). These results suggest that miR-183 down-regulates MTA1 and inhibits the proliferation of NSCLC cells. 
Fig. 6. RT-qPCR results indicate that up-regulation of miR-183 expression decreases the expression of MTA1 mRNA.. Note: RT-qPCR, reverse transcription quantitative polymerase chain reaction; miR183, microRNA-183; NC, negative control; PCNA, proliferating cell nuclear antigen; MTA1, metastasisassociated protein $1 ;{ }^{*}, \mathrm{p}<0.05$ compared with the blank and NC groups.

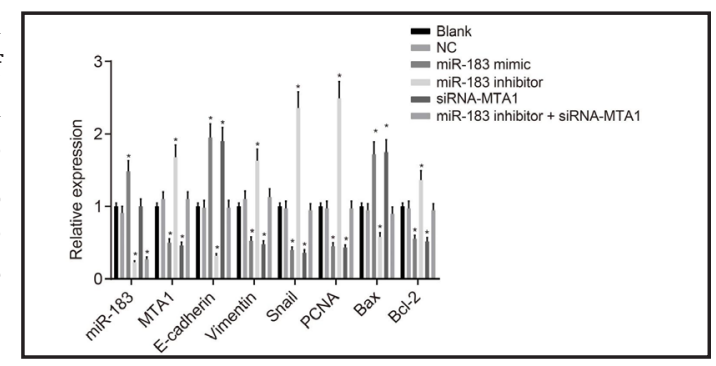

Fig. 7. Western blot analysis results suggest that up-regulation of miR183 expression decreases the expression of MTA1 protein.. Note: $\mathrm{NC}$, negative control; A, histogram analysis of protein expressions; B, electrophoregrams of proteins; PCNA, proliferating cell nuclear antigen; MTA1, metastasis-associated protein 1; *, $\mathrm{p}<0.05$ compared with the blank and NC groups.

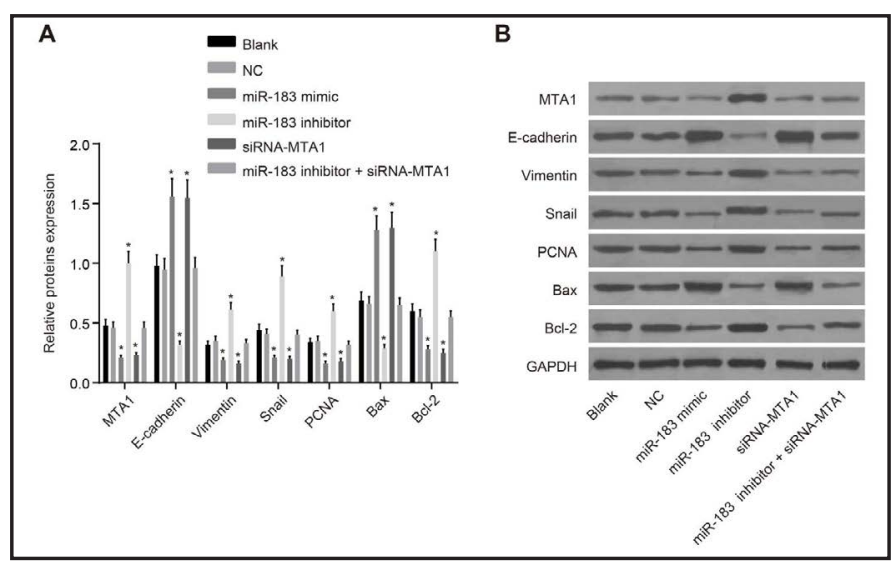

Up-regulation of miR183 arrests cells in the G0/G1 phase

Next, we studied the effect of miR-183 on the NSCLC cell cycle using the flow cytometry assay. The proportion of cells in the G0/G1 phase in the blank, NC, miR-183 mimic, miR183 inhibitor, siRNA-MTA1,

Fig. 8. CCK8 results suggest that upregulation of miR183 inhibits NSCLC cell proliferation. Note: NC, negative control; CCK8, cell counting kit-8; OD, optical density; *, $\mathrm{p}<0.05$ compared with Blank and NC groups after $24 \mathrm{~h}$. and miR-183 inhibitor + siRNA-MTA1 groups were $49.12 \pm 0.25 \%, 48.87 \pm 0.48 \%, 61.99 \pm 0.89 \%, 22.30 \pm 1.20 \%, 60.12 \pm 4.58 \%$ and $48.55 \pm$ $0.33 \%$, respectively, and the percentages of cells in the $\mathrm{S}$ phase were $40.04 \pm 1.27 \%, 40.22$ $\pm 0.78 \%, 24.62 \pm 1.54 \%, 66.00 \pm 0.77 \%, 23.49 \pm 3.57 \%$ and $40.00 \pm 0.89 \%$, respectively. There was no significance difference in the proportion of cells in the $\mathrm{S}$ phase between the blank and NC groups $(p>0.05)$. Compared with the blank and NC groups, the proportion of cells in the G0/G1 phase increased significantly, and the proportion of cells in the S phase decreased in the miR-183 mimic and siRNA-MTA1 groups. The miR-183 inhibitor group exhibited an opposite trend (all $p<0.05$ ). No significant difference was observed in the miR183 inhibitor + siRN-MTA1 group $(p>0.05)$. The proportion of cells in the G2 phase among all groups exhibited no remarkable difference $(p>0.05)$ (Fig. 9). These data suggest that overexpression of miR-183 can shorten the NSCLC cell cycle.

\section{Up-regulation of miR-183 promotes NSCLC cell apoptosis}

We also investigated the effect of miR-183 on NSCLC cell apoptosis using flow cytometry. There was no significant difference in the apoptotic rate between the blank and NC groups $(p>0.05)$. Compared with the blank and NC groups, the apoptotic rate in the miR-183 inhibitor group decreased significantly, while that in the miR-183 mimic and siRNA-MTA1 
Fig. 9. Flow cytometry results indicate that up-regulation of miR-183 inhibits NSCLC cell cycles.. Note: A, cell cycle using flow cytometry; B, percentage of cell cycle; NC, negative control; miR-183, microRNA-183; MTA1, metastasis-associated protein $1 ;{ }^{*}, \mathrm{p}<0.05$ compared with the blank and NC groups.

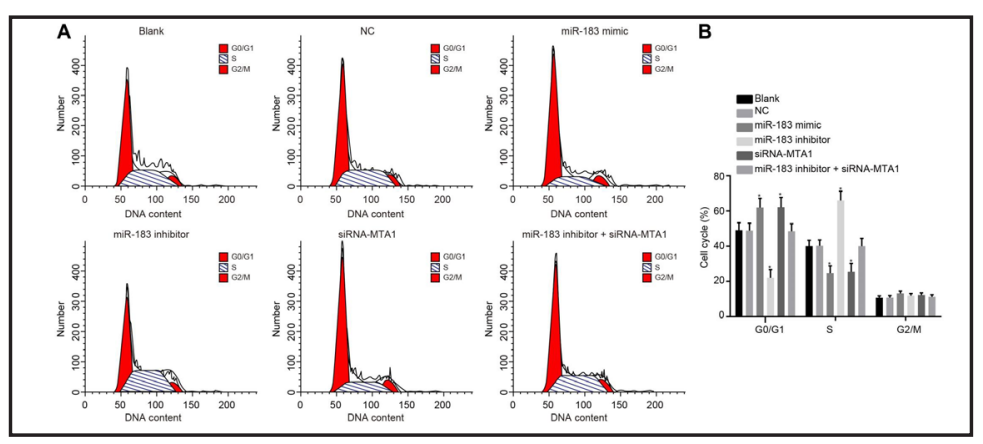

Fig. 10. Flow cytometry results indicate that up-regulation of miR-183 promotes NSCLC cell apoptosis. Note: A, cell apoptosis among six groups; $\mathrm{B}$, the apoptosis rate of six groups; NC, negative control; miR-183, microRNA-183; MTA1, metastasis-associated protein $1 ;{ }^{*}, \mathrm{p}<0.05$ compared with the blank and NC groups.

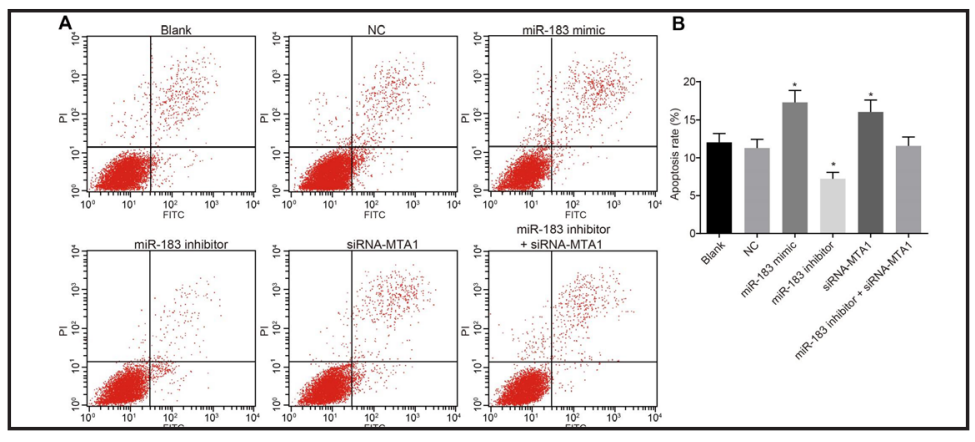

groups increased (all $p<0.05$ ). Furthermore, no significant difference in the apoptotic rate was observed in the miR-183 inhibitor + siRN-MTA1, blank and NC groups ( $p>0.05)$ (Fig. 10). These data reveal that overexpression of miR-183 can promote NSCLC cell apoptosis.

Up-regulation of miR-183 inhibits NSCLC cell migration and invasion

To investigate the effects of miR-183 on NSCLC cell migration and invasion, the scratch test and Transwell assays were performed. The results are shown in Fig. 11. The trends of cell migration and invasion in each group were the same. The blank and NC groups produced no notable difference in cell migration and invasion ability $(p>0.05)$. Compared with the blank and $\mathrm{NC}$ groups, the migration and invasion abilities of the cells were significantly decreased in the miR-183 mimic and siRNA-MTA1 groups, while they were increased in the miR-183 inhibitor group (all $p<0.05$ ). In addition, the difference

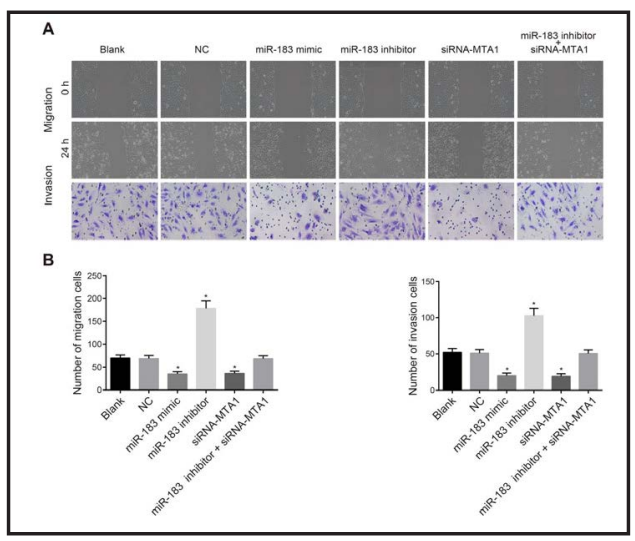

Fig. 11. Results of the scratch test and Transwell assays reveal that up-regulation of miR-183 inhibits the migration and invasion of NSCLC cells. Note: NC, negative control; NSCLC, non-small cell lung cancer; $A$, migration and invasion ability of cells under a microscope; $\mathrm{B}$, the number of migration and invasion cells; *, $\mathrm{p}<0.05$ compared with blank and NC groups. in the cell migration and invasion ability in the miR-183 inhibitor + siRN-MTA1 group was statistically insignificant $(p>0.05)$. The results suggest that overexpression of miR-183 inhibits NSCLC cell migration and invasion.

Up-regulation of miR-183 inhibits tumor size and growth rate of NSCLC

Finally, we evaluated the effects of miR-183 on tumor size and tumor growth rate of NSCLC using tumor xenografts in nude mice. The results indicate that there are no significant differences in tumor size and tumor growth rate between the blank and NC groups $(p>0.05)$. 


\section{Cellular Physiology Cell Physiol Biochem 2018;46:93-106 \begin{tabular}{l|l} 
DOI: 10.1159/000488412 & a 2018 The Author(s). Published by S. Karger AG, Basel \\
www.karger.com/cpb
\end{tabular} \\ Yang et al.: Role of MiR-183 and MTA1 in NSCLC}

Subcutaneous tumors in the miR-183 inhibitor group were significantly larger than in the blank and NC groups, and the tumor growth rate was the highest ( $p$ $<0.05$ ). Tumor size and tumor growth rate in the miR-183 mimic and siRNAMTA1 groups were significantly lower than those in the blank and NC groups $(p<0.05)$. No significant difference was observed in the miR-183 inhibitor + siRNMTA1 group ( $p>0.05$ ) (Fig. 12). These results indicate that overexpression of miR-183 can down-regulate MTA1 and inhibit the tumor size and growth rate of NSCLC.

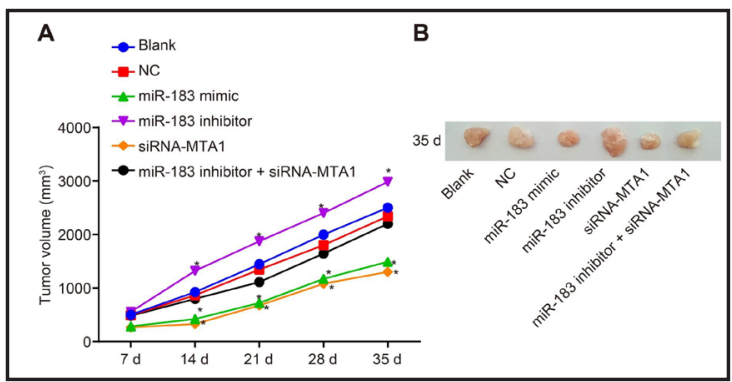

Fig. 12. Results of tumor xenografts in nude mice suggest that up-regulation of miR-183 inhibits the tumor volume (A) and size (B) of NSCLC. Note: NC, negative control; ${ }^{*}, \mathrm{p}<0.05$ compared with the blank and NC groups.

\section{Discussion}

NSCLC is a malignant tumor that comprises $85 \%$ of lung cancers and is often diagnosed in late stages, leading to poor survival [22]. MiRs increasingly play a potential and core role in the regulation of the malignant development of cancer by acting as oncogenes or tumor suppressors [23]. Furthermore, Lee et al. revealed that overexpression of MTA1 was linked to a higher tumor grade and resulted in poorer clinical outcomes in human breast cancer [24]. In this study, we investigated the effect of miR-183 on the invasion and metastasis of NSCLC by mediating MTA1 and found that the up-regulation of miR-183-induced MTA1 restrains the proliferation, EMT, migration and invasion of human NSCLC cells. As the initial discovery in this study, MTA1 was identified as a potential target gene of miR-183 according to http:// www.microrna.org, which was further verified experimentally by a luciferase reporter gene assay. In addition, miRs may interact with several mRNA targets via perfect or imperfect base pairing, mainly in the 3'-UTR portion [25].

Our results demonstrated that compared with the blank and NC groups, the apoptotic rate in the miR-183 inhibitor group was significantly lower than that in the miR-183 mimic and siRNA-MTA1 groups. Furthermore, the migration and invasion abilities of cells were significantly reduced in the miR-183 mimic and siRNA-MTA1 groups but were increased in the miR-183 inhibitor group. This suggested that the down-regulation of MTA1 could inhibit the proliferation of NSCLC cells and promote cell apoptosis, as well as suppress cell invasion and migration. Furthermore, previous studies have focused on the diagnostic function of miRs between NSCLC patients and normal controls [26-28]. Subsequent receiver operating characteristic (ROC) curve analysis indicated that the miR-183 level could serve as an early diagnostic biomarker for NSCLC, with high sensitivity and specificity [29]. More importantly, overexpression of miR-183 and MTA1 gene silencing significantly reduced proliferation and induced apoptosis of human prostate cancer (PC) cells [13].

Additionally, miR-183 expression and the expression levels of E-cadherin and Bax mRNA and protein were reduced, but the expression levels of MTA1, Vimentin, snail, PCNA and Bcl2 mRNA and protein were elevated. Compared with the blank and NC groups, the expression levels of E-cadherin and Bax mRNA and protein increased, while the expression of MTA1, Vimentin, snail, PCNA and Bcl-2 mRNA and protein decreased in the miR-183 mimic and siRNA-MTA1 groups. This suggested that MTA1 and miR-183 may play an important role in the tumorigenesis, development and metastasis of some cancers, as in NSCLC. It has been reported that the expression of E-cadherin increased in the miR-183 mimic and MTA1-siRNA groups, while the expression of MTA1 and Vimentin were significantly decreased in the miR183 mimic and MTA1-siRNA groups [13]. Moreover, another study has shown that MTA1 
expression is induced in advanced ovarian cancer, which is known to repress ER $\beta$ expression and increase the expression of an oncogenic cytokine growth-regulated oncogene (GRO) [30].

The proportion of cells in the G0/G1 phase was significantly increased, while the proportion of cells in the S phase was decreased in the miR-183 mimic and siRNA-MTA1 groups. The miR-183 inhibitor group exhibited an opposite trend, indicating that upregulation of miR-183 expression could increase the proportion of cells in the G0/G1 phase, lower the proportion of cells in the S phase and inhibit the growth of NSCLC cells, which might suggest that the induction of cell cycle arrest is a significant anti-proliferative mechanism [31]. Another study demonstrated that up-regulation of miR-183 increased the expression and nuclear localization of cyclin D 1, and the down-regulations and nuclear localizations of p21 and p27 caused G1 progression in NSCLC cells [32].

Additionally, our results demonstrated that subcutaneous tumors were larger than those in the blank and NC groups and that the tumor growth rate was highest in the miR183 inhibitor group. The tumor size and tumor growth rate in the miR-183 mimic and siRNA-MTA1 groups were lower than those in the blank and NC groups, suggesting that upregulation of miR-183 and down-regulation of MTA1 may improve the survival time of NSCLC patients. Another study demonstrated that MTA1 could serve as one of the key upstream regulators of tumorigenesis and cancer progression [33]. It is known that patients with high levels of expression of MTA1 exhibited significantly poorer survivals compared with those with low levels of expression of MTA1 [34]. These findings suggested that MTA1 was involved in the modulation of cancer progression and might be useful as a novel potential therapeutic marker.

In conclusion, our preliminary findings confirmed that up-regulation of miR-183 and down-regulation of MTA1 could inhibit cell proliferation, invasion and migration and promote cell apoptosis, which provides a novel approach for the treatment of NSCLC. However, a few limitations remain in the present investigation. Additional investigations with a larger sample size in human populations are required for the application of targeted therapy for NSCLC. We will extend the time-period of our study to involve more subjects and will pursue further research to explore the association between miR-183 and MTA1 in NSCLC cells.

\section{Acknowledgements}

We would like to acknowledge the helpful comments on this paper received from our reviewers.

\section{Disclosure Statement}

We declare that we have no conflicts of interest.

\section{References}

-1 Felip E, Garrido P, Trigo JM, Lopez-Brea M, Paz-Ares L, Provencio M, Isla D, Spanish Society of Medical O: SEOM guidelines for the management of non-small-cell lung cancer (NSCLC). Clin Transl Oncol 2009;11:284-289.

2 Salskov A, Hawes SE, Stern JE, Feng Q, Jordan CD, Wiens L, Rasey J, Lu H, Kiviat NB, Vesselle H: Hypermethylation of CCND2 May Reflect a Smoking-Induced Precancerous Change in the Lung. J Oncol 2011;2011:950140.

- J Jiang SS, Fang WT, Hou YH, Huang SF, Yen BL, Chang JL, Li SM, Liu HP, Liu YL, Huang CT, Li YW, Jang TH, Chan SH, Yang SJ, Hsiung CA, Wu CW, Wang LH, Chang IS: Upregulation of SOX9 in lung adenocarcinoma and its involvement in the regulation of cell growth and tumorigenicity. Clin Cancer Res 2010;16:43634373. 


\section{Cellular Physiology Cell Physiol Biochem 2018;46:93-106 \begin{tabular}{l|l} 
and Biochemistry Published online: March 23, 2018 & $\begin{array}{l}\text { C) } 2018 \text { The Author(s). Published by S. Karger AG, Basel } \\
\text { www.karger.com/cpb }\end{array}$ \\
\hline
\end{tabular}}

Yang et al.: Role of MiR-183 and MTA1 in NSCLC

4 Tan DS, Camilleri-Broet S, Tan EH, Alifano M, Lim WT, Bobbio A, Zhang S, Ng QS, Ang MK, Iyer NG, Takano A, Lim KH, Regnard JF, Tan P, Broet P: Intertumor heterogeneity of non-small-cell lung carcinomas revealed by multiplexed mutation profiling and integrative genomics. Int J Cancer 2014;135:1092-1100.

5 Jiang N, Chen XC, Zhao Y: Analysis of the risk factors for myelosuppression after concurrent chemoradiotherapy for patients with advanced non-small cell lung cancer. Support Care Cancer 2013;21:785-791.

-6 Mauguen A, Pignon JP, Burdett S, Domerg C, Fisher D, Paulus R, Mandrekar SJ, Belani CP, Shepherd FA, Eisen T, Pang H, Collette L, Sause WT, Dahlberg SE, Crawford J, O’Brien M, Schild SE, Parmar M, Tierney JF, Le Pechoux C, Michiels S, Surrogate Lung Project Collaborative G: Surrogate endpoints for overall survival in chemotherapy and radiotherapy trials in operable and locally advanced lung cancer: a re-analysis of metaanalyses of individual patients' data. Lancet Oncol 2013;14:619-626.

7 Chen K, Fan W, Wang X, Ke X, Wu G, Hu C: MicroRNA-101 mediates the suppressive effect of laminar shear stress on mTOR expression in vascular endothelial cells. Biochem Biophys Res Commun 2012;427:138142.

8 Acunzo M, Visone R, Romano G, Veronese A, Lovat F, Palmieri D, Bottoni A, Garofalo M, Gasparini P, Condorelli G, Chiariello M, Croce CM: miR-130a targets MET and induces TRAIL-sensitivity in NSCLC by downregulating miR-221 and 222. Oncogene 2012;31:634-642.

-9 Jang JS, Jeon HS, Sun Z, Aubry MC, Tang H, Park CH, Rakhshan F, Schultz DA, Kolbert CP, Lupu R, Park JY, Harris CC, Yang P, Jen J: Increased miR-708 expression in NSCLC and its association with poor survival in lung adenocarcinoma from never smokers. Clin Cancer Res 2012;18:3658-3667.

10 Zhang L, Quan H, Wang S, Li X, Che X: MiR-183 promotes growth of non-small cell lung cancer cells through Fox01 inhibition. Tumour Biol 2015;36:8121-8126.

11 Wang G, Mao W, Zheng S: MicroRNA-183 regulates Ezrin expression in lung cancer cells. FEBS Lett 2008;582:3663-3668.

12 Xu F, Zhang H, Su Y, Kong J, Yu H, Qian B: Up-regulation of microRNA-183-3p is a potent prognostic marker for lung adenocarcinoma of female non-smokers. Clin Transl Oncol 2014;16:980-985.

13 Lin X, Zheng L, Song H, Xiao J, Pan B, Chen H, Jin X, Yu H: Effects of microRNA-183 on epithelialmesenchymal transition, proliferation, migration, invasion and apoptosis in human pancreatic cancer SW1900 cells by targeting MTA1. Exp Mol Pathol 2017;102:522-532.

14 Zhu X, Zhang X, Wang H, Song Q, Zhang G, Yang L, Geng J, Li X, Yuan Y, Chen L: MTA1 gene silencing inhibits invasion and alters the microRNA expression profile of human lung cancer cells. Oncol Rep 2012;28:218224.

15 Luo H, Li H, Yao N, Hu L, He T: Metastasis-associated protein 1 as a new prognostic marker for solid tumors: a meta-analysis of cohort studies. Tumour Biol 2014;35:5823-5832.

16 Li SH, Tian H, Yue WM, Li L, Li WJ, Chen ZT, Hu WS, Zhu YC, Qi L: Overexpression of metastasis-associated protein 1 is significantly correlated with tumor angiogenesis and poor survival in patients with early-stage non-small cell lung cancer. Ann Surg Oncol 2011;18:2048-2056.

17 Li S, Tian H, Yue W, Li L, Gao C, Si L, Li W, Hu W, Qi L, Lu M: Down-regulation of MTA1 protein leads to the inhibition of migration, invasion, and angiogenesis of non-small-cell lung cancer cell line. Acta Biochim Biophys Sin (Shanghai) 2013;45:115-122.

18 Moreau D, Jacquot C, Tsita P, Chinou I, Tomasoni C, Juge M, Antoniadou-Vyza E, Martignat L, Pineau A, Roussakis C: Original triazine inductor of new specific molecular targets, with antitumor activity against nonsmall cell lung cancer. Int J Cancer 2008;123:2676-2683.

19 Chen L, San Antonio B, Yan Y, Chen J, Goodloe RJ, John WJ: Safety profiles of non-small cell lung cancer patients treated with pemetrexed plus carboplatin: a real-world retrospective, observational, cohort study. Curr Med Res Opin 2017;33:931-936.

20 Song YM, Lian CH, Wu CS, Ji AF, Xiang JJ, Wang XY: Effects of bone marrow-derived mesenchymal stem cells transplanted via the portal vein or tail vein on liver injury in rats with liver cirrhosis. Exp Ther Med 2015;9:1292-1298.

21 Ayuk SM, Abrahamse H, Houreld NN: The role of photobiomodulation on gene expression of cell adhesion molecules in diabetic wounded fibroblasts in vitro. J Photochem Photobiol B 2016;161:368-374.

-22 Zhu J, Zhang Y, Yang X, Jin L: Clinical Significance and Tumor-Suppressive Function of miR-516b in Nonsmall Cell Lung Cancer. Cancer Biother Radiopharm 2017;32:115-123. 


\section{Cellular Physiology Cell Physiol Biochem 2018;46:93-106

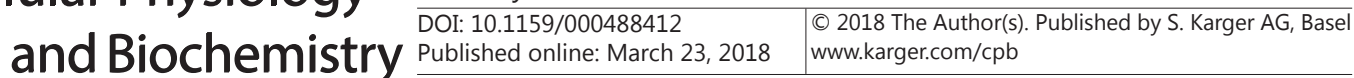

Yang et al.: Role of MiR-183 and MTA1 in NSCLC

23 Kong X, Li G, Yuan Y, He Y, Wu X, Zhang W, Wu Z, Chen T, Wu W, Lobie PE, Zhu T: MicroRNA-7 inhibits epithelial-to-mesenchymal transition and metastasis of breast cancer cells via targeting FAK expression. PLoS One 2012;7:e41523.

24 Lee MH, Koh D, Na H, Ka NL, Kim S, Kim HJ, Hong S, Shin YK, Seong JK, Lee MO: MTA1 is a novel regulator of autophagy that induces tamoxifen resistance in breast cancer cells. Autophagy 2017;10.1080/15548627.2017.13884761-13.

-25 Hausser J, Landthaler M, Jaskiewicz L, Gaidatzis D, Zavolan M: Relative contribution of sequence and structure features to the mRNA binding of Argonaute/EIF2C-miRNA complexes and the degradation of miRNA targets. Genome Res 2009;19:2009-2020.

-26 Mo D, Gu B, Gong X, Wu L, Wang H, Jiang Y, Zhang B, Zhang M, Zhang Y, Xu J, Pan S: miR-1290 is a potential prognostic biomarker in non-small cell lung cancer. J Thorac Dis 2015;7:1570-1579.

27 Chen X, Hu Z, Wang W, Ba Y, Ma L, Zhang C, Wang C, Ren Z, Zhao Y, Wu S, Zhuang R, Zhang Y, Hu H, Liu C, Xu L, Wang J, Shen H, Zhang J, Zen K, Zhang CY: Identification of ten serum microRNAs from a genomewide serum microRNA expression profile as novel noninvasive biomarkers for nonsmall cell lung cancer diagnosis. Int J Cancer 2012;130:1620-1628.

28 Chen X, Ba Y, Ma L, Cai X, Yin Y, Wang K, Guo J, Zhang Y, Chen J, Guo X, Li Q Li X, Wang W, Zhang Y, Wang J, Jiang X, Xiang Y, Xu C, Zheng P, Zhang J, Li R, Zhang H, Shang X, Gong T, Ning G, Wang J, Zen K, Zhang J, Zhang CY: Characterization of microRNAs in serum: a novel class of biomarkers for diagnosis of cancer and other diseases. Cell Res 2008;18:997-1006.

29 Zhu W, Zhou K, Zha Y, Chen D, He J, Ma H, Liu X, Le H, Zhang Y: Diagnostic Value of Serum miR-182, miR183, miR-210, and miR-126 Levels in Patients with Early-Stage Non-Small Cell Lung Cancer. PLoS One 2016;11:e0153046.

-30 Dannenmann C, Shabani N, Friese K, Jeschke U, Mylonas I, Bruning A: The metastasis-associated gene MTA1 is upregulated in advanced ovarian cancer, represses ERbeta, and enhances expression of oncogenic cytokine GRO. Cancer Biol Ther 2008;7:1460-1467.

-31 Hong JY, Chung HJ, Bae SY, Trung TN, Bae K, Lee SK: Induction of Cell Cycle Arrest and Apoptosis by Physcion, an Anthraquinone Isolated From Rhubarb (Rhizomes of Rheum tanguticum), in MDA-MB-231 Human Breast Cancer Cells. J Cancer Prev 2014;19:273-278.

-32 Kang J, Kim W, Lee S, Kwon D, Chun J, Son B, Kim E, Lee JM, Youn H, Youn B: TFAP2C promotes lung tumorigenesis and aggressiveness through miR-183- and miR-33a-mediated cell cycle regulation. Oncogene 2017;36:1585-1596.

33 Dhar S, Kumar A, Zhang L, Rimando AM, Lage JM, Lewin JR, Atfi A, Zhang X, Levenson AS: Dietary pterostilbene is a novel MTA1-targeted chemopreventive and therapeutic agent in prostate cancer. Oncotarget 2016;7:18469-18484.

34 Yuan T, Zhang H, Liu B, Zhang Q, Liang Y, Zheng R, Deng J, Zhang X: Expression of MTA1 in nasopharyngeal carcinoma and its correlation with prognosis. Med Oncol 2014;31:330. 